\title{
Exploration and Practice on the Teaching Path of Critical Thinking Ability in College English Based on Cooperative Learning Theory
}

\author{
Xiaojing Li \\ Zhejiang Yuexiu University, Shaoxing, China \\ Email: 20131050@zyufl.edu.cn
}

How to cite this paper: Li, X.J. (2021) Exploration and Practice on the Teaching Path of Critical Thinking Ability in College English Based on Cooperative Learning Theory. Open Access Library Journal, 8: e7544.

https://doi.org/10.4236/oalib.1107544

Received: May 18, 2021

Accepted: July 9, 2021

Published: July 12, 2021

Copyright $\odot 2021$ by author(s) and Open Access Library Inc.

This work is licensed under the Creative Commons Attribution International License (CC BY 4.0).

http://creativecommons.org/licenses/by/4.0/

\begin{abstract}
Integrated English is one of the compulsory courses in colleges, and combining critical thinking cultivation with the course is an inevitable tendency. Based on Cooperative Learning Theory, this paper aims to analyze the corresponding strategies of cultivating critical thinking and its assessment approaches, so as to pave the way for effective teaching of critical thinking for Chinese university students.
\end{abstract}

\section{Subject Areas}

Language Education

\section{Keywords}

Cooperative Learning, Integrated English, Critical Thinking

\section{1. 引言}

思辨是解释、分析、评价、推断以及对判断赖以存在的论据、概念、方 法、标准或语境的说明。思辨能力, 又称 “批判性思考” 能力, 主要指分析 性与评价性思考, 具有自我调节、自我监控、自我纠错等特点, 可以帮助学 习者通过对信息、结论或者视角的不断质疑, 形成深入思考的能力 [1]。思辨 能力主要培养主观能动性、收集、分析和阐释信息的能力、清晰和准确地提 出关键性问题的能力、得出有理有据的结论和解决问题的能力、对不同思想 和观点持有开放和包容的态度、有效与他人沟通的能力 [2]。将思辨能力的培 养融入大学《综合英语》课程, 能让学生在学习和掌握英语语言技能的同时, 开拓国际视野, 培养科学的思维习惯和发现、解决问题的能力, 培养跨文化 能力和文化思辨能力, 培养创新精神, 提升创新能力。因此, 在英语教学中 
对大学生进行思辨能力的培养极其重要。

\section{2. 大学《综合英语》教学存在的主要问题}

笔者在多年的大学《综合英语》教学实践和教学反思过程中, 发现如下 问题:

1) 教材编写和教学实际使用有一定时滞性, 与学生思辨能力培养相关的 篇幅相对较少。大学《综合英语》教材涵盖大量词汇、句法、语法等语言基 础知识, 较少涉及对篇章的整体和全面把握, 缺少对作者思想的深入理解、 分析和总结。

2) 大部分教师主要围绕语言知识点和文章基本内容进行课程设计。教学 内容同质化, 教学方法雷同的现象比较突出。为完成教学任务、达成教学目 标, 教师花费大量时间和精力向学生灌输课文主题所弘扬的主流价值观, 引 导学生学习、欣赏和模仿课文结构和语言特色。

3）学生在长期应试教育影响下形成了思维定势, 习惯遵循课本内容寻找 “标准答案”, 缺乏质疑精神。学生主要进行浅层思考, 专注于对特定信息 的查找, 缺少思辨能力训练。学生上课时缺乏足够的思考时间和推理空间, 很少辩论、集体深入讨论和思考教学内容, 缺乏自主或集体协作探索和挑战。 学习目的是追求高分数和高绩点, 课下仍然机械地大量刷题, 独立思考能力、 创新能力没有得到充分培养。学生惰于思考, 当然也没有鲜明思想和独特主 张。

\section{3. 提高学生思辨能力的举措}

1）教师应通过多种途径学习思辨能力的相关理论, 参加相关研修项目和 讲座, 加强与专家、同行之间的学术交流。在学校原有基础上搭建思辨能力 培养的教学资源共享和交流平台。教师应积极提高自身思辨水平，只有对中 西方国家文化有深层次理解后，才能理性对比和分析中西方文化差异，提高 自身的跨文化思辨能力, 继而在教学实践中通过案例、讲故事、情景剧等形 式向学生传授跨文化知识, 通过讨论、辩论、展示等方式帮助学生从更深层 次理解文化差异, 从而提高学生的跨文化思辨能力。

2) 教师应努力构建新型教学模式, 有计划、按步骤地引入思辨能力教学 策略和方法, 深入探讨与分析《综合英语》教学课程内容, 明确思辨能力教 学目标, 灵活高效地设计课程。

教师应根据当前教学内容合理设置所提问题, 营造平等、轻松的课堂氛 围和思辨环境, 采取多种措施鼓励学生勤思善辨。学生有了疑问, 如教师加 以合理引导, 自然会形成思辨, 并在个人和群体思辨过程中突破传统思维定 势，顺其自然就会形成创新。

教师应经常评估和反思自身教学，根据具体情况不断改进教学策略和方 法, 以培养学生缜密的逻辑思维习惯。在课程目标和内容设计上, 不仅全面 涵盖课程知识点更要重点培养学生的思辨能力。通过科学有效的课程设计练 习逐步提高大学生的元思辨能力和自我反思能力。 
教师对学生思维能力进行训练、检查、调整和评估, 使学生主观能动性 得以充分发挥。传统大学英语课堂教师预设教学内容, 学生通过反复刻意练 习来巩固所学知识; 在培养思辨能力的课堂, 突出 “学生中心”、“小组合 作”、“真实语境”, 兼顾英语语言与思维的训练。教师应指导学生掌握合 理提问策略, 鼓励个人独立思考和学习小组协同配合进行 “头脑风暴” , 引 导学生发现并解决问题, 让学生在实践中发展认知, 提升思辨能力。

教师应在大学《综合英语》教学的各环节中创造性地运用思辨的理念和 技能。课前预习提出问题, 课堂 “精读” 解决问题。学习小组分工合作形成 评判, 通过多轮提问补充语言知识和可理解性的输入, 师生、生生评价激发 学生的语言输出技能。在环环相扣、目标明确的 “训练式” 教学过程中, 培 养学生反思质疑的能力和依据标准评价思维的技能。

3) 教师在课前预习环节将学生按寝室分成多个学习小组, 事先明确预习 内容, 甄选出课文的重点语句、词汇、短语、精彩篇章和有疑义、需要讨论 的问题, 要求并鼓励学习小组根据事先的任务分工完成相关预习任务, 由寝 室长(或小组长)记录预习的重点知识和内容。

也可以采取师生共同参与课程建设, 共同参与篮选单元主题内容的方法。 让学生挑选课本上感兴趣的单元作为教学内容, 或根据大部分同学的意见, 与学生商定下一讲的具体内容。课堂活动以小组分工与合作形式开展, 学习 小组成员之间针对预习内容进行合作、互动和讨论, 不同学习小组之间进行 头脑风暴、沟通和辩论, 学生从而能获得多方面的信息, 知识、观点、情感 得到有效交流。

通过学习小组分工合作, 学生学会倾听不同观点和意见, 培养多视角、 多方位思考问题的能力, 分析思考和解决问题的能力也能相应提高。学生的 学习积极性和主观能动性空前高涨, 兴趣大增。因而能充分发挥主观能动性, 激发学生灵活运用既有储备知识、生活经验和价值观念进行独立思考、理智 分析并实施独立判断。

教师鼓励学习小组分工合作寻找课外补充材料, 培养学生搜集、分析、 阐释、分享信息、相互沟通的能力。按照思辨能力层级理论, 认知技能是思 辨能力的主要表现形式。提升认知技能必须是以问题为出发点, 通过不断的 讨论与交流, 才能丰富自己的认知, 养成开放、包容、自信等人格特质 [3]。 小组活动中学生同伴之间在交流中可以互相借鉴, 学会从不同角度看待问题, 并从同伴中获得情感支持, 因而心理负担较小, 可以做到畅所欲言。在组内 和组间讨论中虽有批判性反馈, 但应遵循不能随意评判他人的准则, 这样学 生逐渐能学会倾听他人, 容忍、尊重不同的意见。学生监控自己的思维过程、 不断调整与反思, 也有助于思辨人格倾向的培养, 养成认真、谦逊、正直、 公正的好品德。

4) 每一堂课教师都应设计 “提问和讨论” 环节, 促使学生独立思考、小 组讨论、分析报告, 最后教师进行总结和点评。课堂上, 教师针对相关话题 提出正面与反面、现在与过去、国内与国外等多角度的问题, 引导学生深思 善辩。比如教师列举一些中西方文化比较的例子, 通过头脑风暴式的讨论使 学生掌握如何用英语讲述中国文化, 了解如何在不同文化背景下避免跨文化 
的冲突。正如 “美国梦” 和 “中国梦” 的区别, 西方文化中对个人主义的极 致推崇甚或影响我国学生理想信念的养成, 而 “中国梦” 兼顾个人发展、国 家强大与民族复兴, 学生在追求个人梦想实现的同时也要体现责任意识、对 社会的贡献、集体主义等, 两者在价值观和终极目标上不可同日而语; 教师 引导学生正确看待我国高新技术企业(如华为公司 5G 设备被西方国家禁用等) 在走出国门过程中遭受的诸多不公平待遇。中国人对西方的传统印象是高度 文明、科技发达、文化包容、充满道德优越感、创造力强大的社会, 而如今 国人对于西方的印象则是粗鲁、恃强凌弱、爱甩锅、充满混乱和骄傲自大; 西方社会对于中国社会的刻板印象是麻木的、不自由的、粗鄙落后的、尚武 且喜好扩张的、爱偷东西和集权化大一统的。中西方对于彼此的认知充满了 不信任和怀疑, 文化壁垒使得双方难以取得有效沟通。基于此, 教师应指导 学生客观分析国内、国际的问题, 理性看待中国在发展过程中出现的危机和 遭遇的困难, 鼓励学生深入探讨和思辨, 正面引导学生的世界观和价值观。

教师依据文本, 精心设计, 善于提出问题, 着力培养学生的问题意识。 教学以问题为导向, 提出问题, 思考问题, 解决问题。因为开放性问题没有 明确答案, 为学生预留下广阔的思考空间。学生能敏锐感受作者想要表达的 思想和含义, 明确作者文字的言外之意。学生能灵活应用既有知识, 通过综 合类比、探索推理、总结归纳等方式得出结论。

教师提问时应合理分配低阶和高阶问题的比例。低阶问题具封闭性与确 定性, 回答缺少互动且停留在事实性或客观性层面, 学生能按图索瀷寻找所 谓的 “标准答案” , 语言输出量有限; 而高阶问题则以参考性问题为主, 具 开放性、不规则与复杂性, 解决方案趋于多样化, 能引导学生输出结构更为 复杂的语句 $[4]$ 。

教师提问时应注意传递积极和正向的强化信息, 保持点头、眼神接触、 附和、肯定和言语鼓励, 根据学生反应灵活把握候问时间, 尽量避免自问自 答。针对教学内容的分析和讨论, 师生、生生之间应有效积极互动, 学生积 极主动思考、讨论、学习、提问、评价和反思。师生互动讨论问题时, 教师 应多肯定, 指出错误与不足时要讲究方式方法, 尽量保持态度亲切、语气适 度, 营造轻松愉快、循循善诱的学习氛围, 让学生彻底放下思想包袱。

a) 在课堂教学环节导入阶段, 教师指定学习小组以寝室为单位在课前提 前预习教学内容, 学生有充足时间搜集相关素材和论据。课堂上学习小组在 组内分享讨论彼此的观点, 通过学习小组背景知识汇报与现场自我评价、教 师现场提问与评价、学生相互提问与评价, 学生能在互动交流中提高反思、 质疑和评判技能等思辨能力。如某学习小组做完课文背景知识介绍后, 教师 从材料的相关性、语言组织的条理性和逻辑性、语言表述的通俗流畅程度等 做相关评价。

b) 在课文讲解阶段, 教师通过针对文章结构模式的提问, 鼓励学生理清 文章结构逻辑顺序并概括主题。通过详细解析重点词汇、语句和句型, 在文 章段落、篇章结构、寻找关联、综合对比和概括等方面引导学生开展提高思 辨能力的训练。教师提出开放式问题后, 各学习小组组内和组间通过分析、 综合、阐释、比较、关联、分类、推理、概括等思辨技能进行积极主动的自 
主探究。教师应鼓励学生质疑, 发展学生的逆向思维能力, 养成多视角思考 问题的习惯。

c) 在语言讲解阶段, 优先解决语言知识理解等重点、难点问题。采取启 发式教学, 和学生充分互动, 让学生独立思考遇到的问题。可采取情景剧表 演形式, 按学习小组分工合作, 指定各学习小组分析故事主人公行为背后的 假设, 给出合理的检验假设的建议, 鼓励学生从不同角度解读具体情境, 提 出多种假设并让学生通过情景剧表演来检验这些假设。

d) 在学生评价阶段, 教师引导学生以学习小组为单位复述课文并讲述对 课文的个人理解(包括最精彩的段落、值得深思的部分和感悟最深的内容)。 在学生互评中, 教师给定相关评判标准, 如复述的内容是否契合课文主题、 思路清晰程度、语言准确和精炼程度、个人观点的深度等。

在模拟课堂教学方法中, 学习小组对任务进行分工, 模拟教师的提问方 法、篇章分析和评价方法, 完成篇章结构分析、语言特色和写作手法的赏析, 呈现文章精华部分。找寻文本特色, 能增强学生概括能力; 其它学习小组对 人物形象、主题和作者的情感经历进行个人或小组评价。

5) 学生成绩评价体系纳入学生思辨能力的考查。如在阅读测试中设计阅 读文本的开放式论述题。设计多种需要运用分析、推理、归纳、创新等思维 活动的拓展类活动, 并提高过程性评价的分值比例。如开展案例分析、小组 讨论和辩论、个人或小组演示、个人或小组调研项目、调查报告、阅读报告 等活动, 学生的活动表现纳入期末学生成绩评价体系。教师应设计评价表和 反馈表, 根据学生语言流利度和课堂参与度、表达内容的广度和深度、独立 思考能力和质疑的能力、逻辑的严密性、内容的原创性等给出评价, 通过访 谈、个案研究、调查问卷等多种方式对学生的思辨能力进行评价。

6) 鼓励学生在课外阅读。教师应提供与课堂阅读主题相关的阅读材料, 开阔学生的思维和视野, 帮助他们熟练掌握并灵活运用多种阅读方式。也可 提供与课堂阅读主题观点对立的阅读材料, 鼓励学生善于思考、敢于评价, 深入分析和独立评价阅读文本。

7) 教师设计主题式写作任务, 学生完成写作任务之后, 通过学生自评、 生生互评、教师评价等, 从不同角度为学生提出修改意见。

课文学习完成后, 教师布置撰写学习笔记的任务。学生根据同学和教师 的意见, 修改并完善学习笔记, 明确优势和不足, 学会并善于反思。

8) 教师深度研究、分析学生学习特点和学习进度, 结合学校实际开展英 语角、英语寝室、英语演讲、英语辩论、分级阅读打卡、写作比赛等一系列 提高学生思辨能力的课外活动。

9) 从学生的角度来说, 课前应提前了解东、西方文化和思想差异, 增加 与学习主题相关的信息量和词汇量, 扩大知识面。不拘泥于学习材料的知识 与观点, 灵活运用思维进行思辨; 在课堂学习中开动大脑、积极思考、学会 反思、总结学习。积极发现并解决问题, 在实践中感悟所学, 逐步提升认知 储备和思辨能力。养成撰写学习笔记的习惯, 根据师生、生生评价的建议修 改自己创作的文章; 在小组活动中学会倾听不同观点和意见, 从不同视角、 多方位地思考问题, 敢于提出疑问并质疑, 敢于提出并佐证自己的独特观点, 
敢于挑战传统、固有观念和权威; 课后不断训练、检查、调整和评估自己的 思维能力, 充分调动自身的主观能动性。

\section{4. 改进评测方法}

当前的课程评测在培养学生的思辨能力方面存在以下不足：测评方式单 一, 测评内容的思辨性不足, 思辨能力测评标准缺失, 形成性评估权重较低。

\section{1. 丰富评测形式和内容}

从纵向来看, 对学生思辨能力的评价应贯穿于学生学习的全过程; 从横 向来看, 教师应从听力、口头表达、阅读和写作等角度考察学生的英语能力; 从思辨深度来看, 应从学生认知、情感、意志、个性等认知发展水平角度来 评价学生的学习情况。

在传统客观测试题型基础上，增加主题性小论文撰写等主观测试题型的 比例。除笔试外, 增加小组讨论和辩论、个人或小组演示、读书报告、个人 或小组调研项目等整体评价方式。目前笔者试行的学生学业成绩评测分数比 例为: 笔试 $(50 \%)$ 、小组讨论和辩论 $(10 \%)$ 、个人或小组演示 $(10 \%)$ 、读书报告 (10\%)、个人或小组调研项目 $(10 \%)$ 、学术性论文 $(10 \%)$ 。

师生共同参与评价。学生不仅是评价的主体和客体, 也要对自己和同学 的学习情况进行评价。教师统计自评、互评分数并收集学习小组的意见和建 议, 在课堂教学结束后统一给学生作反馈, 鼓励并指导学习小组进一步完善 小组作业。

\section{2. 增强评测思辨性}

减少终结性评估权重, 加大形成性评估比例和分值。形成性评估由学习 小组调研项目和个人项目学术小论文组成。学习小组项目要求学生分组后自 选题目, 进行资料收集和课外调研, 学习小组成员轮流在课堂上口头汇报成 果。小组成员依据成果从不同角度切入主题, 独立撰写、提交个人研究课题 的小论文。项目初始阶段, 学习小组成员从教师指定的选题目录中自主确定 选题方向, 在查阅相关文献资料的基础上最终确定小组研究题目。学习小组 分工合作建构发言大纲, 其内容包括文献综述、背景知识综述, 个人观点和 见解汇总。学生在此过程中不仅要搜集海量信息, 对其进行整理、分析、归 纳、总结、评价, 还要构建思路清晰、逻辑严密的主题框架结构。学习小组 口头陈述成果后, 生生互动提问和答辩。口头展示后学生开始独立撰写相关 小论文，提出问题并分析、论证观点。学生在进行大量此类练习后，对信息 的理解、运用、分析和评价能进一步达到高层级思辨阶段。学生针对教学内 容、阅读材料写评论文章，能培养分析、判断、对比、阐释、表达、解决问 题的能力。

课程的终结性评估采用学术性论文写作的方式。定题环节中, 学生深刻 理解、分析、比较、归纳总结论文相关信息, 形成个人独特见解, 确立有思 考深度的中心论点。随后学生厘清所写论文的基本框架、观点阐述、中心论 点与分论点及分论点之间的逻辑关系。通过比较和分析数据, 最终选取相关 
的、典型的和有说服力的论据来完成论证过程。

\section{3. 明确思辨能力评测标准}

从认知标准入手，从思维的清晰性、相关性、逻辑性、深刻性和灵活性 五个角度来评价学生的思维能力。论文在思辨方面的评判标准要求观点鲜明, 论证有力, 内容充实, 有明确的独立观察和思考内容, 有较强的批判和研究 能力。引导学生针对具体情境有意识地启动思维能力, 充分发挥其主观能动 性。考察学生是否有好奇、开放、自信等思维情感特质; 考察其对提出的论 题能否提出有说服力的论据, 论据越多则相应分值越高; 对别人的观点能否 做出恰当评论并质疑; 考察其对文本内容能否提出深层次问题; 能否补充他 人观点并说明理由; 能否总结他人观点; 能否找出并验证假设等, 将其一并 纳入形成性考核评价成绩。

\section{4. 丰富评测结果的呈现方式}

可以采取 “图示” 评测结果的形式取代传统的分数作为评价结果的呈现。 如将 “雷达图” 作为评测结果的一部分, 呈现学生各项英语思辨能力的横向 对比, 更直观地看出学生多向度知识或者能力的成长情况; 或把 “长条图” 纳入评测结果，将目标达成的进度在长条图中呈现出来。

\section{5. 基于小组合作理论的大学《综合英语》思辨能力培养课堂 教学实践}

以下就新目标大学英语《综合教程 1》Life at Harvard University一文为 例, 从思辨能力培养角度进行教学设计。

\section{1. 课前创设情景}

根据主题 - 大学生活, 教师课前在教学平台上分享相关文本、音视频等 多模态资源并提出思考问题 “Are there any differences between college and high school? List some examples to support your ideas.”。学生以小组为单位在 线上讨论区进行交流, 预热单元话题。

\section{2. 课上师生合作交流，展示成果}

\subsection{1. 课前启发环节}

教师引导学生回顾“What were your expectations of your college life before coming to your university?”启发学生分享对大学生活的认知。

\subsection{2. 课堂讨论与展示}

在前期话题讨论的基础上, 布置小组任务 “Ideal College Life in Our Eyes”。帮助学生在充分交流展示后, 进一步深入文章主题。

\subsection{3. 知识构建与思辨培养}

1) 教师以灵活多样的形式帮助学生进行文化背景知识构建, 提高人文素 
养。如设置相关背景知识的判断正误题、连线匹配题等。

如 About American Education (True/False):

a) Education is governed by state and local governments instead of the national government in America.

b) Yale University is the oldest institution of higher education in the United States.

c) All American children are offered 12 years of compulsory public education.

d) Some Parents select home schooling to educate their children due to the moral or religious reasons.

e) Harvard University is a public Ivy League research university in Cambridge, Massachusetts, established in 1636.

f) American education puts more emphasis on developing critical thinking rather than acquiring quantities of facts.

2) 在拓展语言点的使用环节, 教师应尽量呈现与当前时事结合的语料。 引导学生借助文中有用表述如 burst into tears, have the drive to do 从自身经 历、当下疫情和中国文化等方面出发, 对课文内容进行思想层面的深入探讨, 帮助学生树立心怀感恩、服务国家的意识和情怀。

3) 以下重点呈现文章分析环节。通过多样的教学活动, 教师多角度培养 学生的思辨能力。重点引领学生认识到哈佛大学这类著名学府也存在其富有 争议的方面, 从而潜移默化地使学生批判性地看待西方文化。

Part 1: 学生通过观看视频 The Ivy League 思考 “What kind of people can enter the Ivy League?” ; “If you were the author, what decision would you make?”。教师组织学生进行小组讨论: “Is the admission system in Harvard perfect? Is American education impeccable? Why or why not?” 帮助学生进一步 了解国外大学真实状态, 客观审视中西方教育的优劣势, 培养求真意识和比 较意识。

Part 2: 教师结合这部分内容抛出互动问题“What did you see and how did feel on the first day of college? Have you encountered any difficulties in your college life so far? If yes, how should you overcome them?”引发学生对文章作 者的情感共鸣, 鼓励学生带入个人情感, 探讨如何发挥工匠精神、攻坚克难、 追求自我。

Part 3: 学生观看视频 College Life of Kelly, 思考 “Is Kylie's college life different from your assumption? If yes, how is it different in details? If no, share us the similarities with yours. What are the things you can learn from Kylie's college life?” 分析中外大学生活的差异, 借鉴西方大学生活有益之处为中国 学生大学生活所用, 培养学生批判性地看待问题的思维方式。

Part 4: 结合文中提及的哈佛裸奔文化, 联系国内外相关新闻报道, 引导 学生思考 “How should we relieve pressures in life?”。小组探讨如何合理应对 生活中的压力及如何辩证看待多元文化。鼓励学生将自己的课余生活与作者 的哈佛生活进行对比, 探讨怎样平衡大学生活, 获得全方面提升。组织学生 
分析扩展阅读文章 Affirmative Dissatisfaction 观点, 回答 “Do the so-called equal rights exist? ”引导学生进一步客观看待西方文化, 通过内隐的方式达 到坚定文化自信的目标。

Part 5: 总结归纳, 升华主题。引导学生思考: “One of the best things Harvard has to offer is its diversity. What does 'diversity' mean here? 结合文 章, 鼓励学生联系当下全球新冠疫情, 思考 “What are we blessed with?”, 得出结论 “We Chinese are always protected by the bravest” ，解读 “大爱” 的 涵义, 融入感恩的育人元素, 进一步增强文化自信及民族自豪感。

\subsection{4. 反思总结}

1) 要求学生观看视频 Why do we go to college? 并总结大学的意义所在。 于平台讨论区展开讨论 “Why should you become a college student with meaningful life?” 巩固课堂学习, 以读促写, 实现知识向能力的转化。

2) 鼓励学生以小组为单位绘制大学生活蓝图。“In groups, make a poster for your shared goals in college: a) What are the goals? b) Why do you all share these goals? c) How are you going to realize them? d) When will you make each of them come true? e) What are the benefits of achieving these goals?” 教师结合 本文作者经历及本校优秀校友的事迹, 激励学生树立正确人生目标, 有效利 用大学时光, 努力实现个人价值。帮助学生既能掌握丰富实用的语言表达, 又能提高思辨能力, 增强人文素养, 切实实现语言与育人的双重目标。

\section{Conflicts of Interest}

The author declares no conflicts of interest.

\section{References}

[1] 马雁. 大学英语课堂有效提问探究——基于思辨能力培养[J]. 海外英语, 2019(7): 103-104.

[2] 夏恒霞. 混合式教学模式下思辨能力的培养一一以学英语课堂为例[J]. 现代交 际, 2019(15): 161-162.

[3] 文秋芳, 等. 构建我国外语类大学生思辨能力量具的理论框架 [J]. 外语界, 2009(1): 37-43.

[4] 王帅. 国外高阶思维及其教学方式[J]. 上海教育研究, 2011(9): 31-34.

\section{Appendix (Abstract and Keywords in Chinese)}

\section{基于小组合作理论的大学《综合英语》思辨能力教学路径探索与实践}

摘要: 大学《综合英语》作为一门大学必修课程, 在其教学实践中加强 思辨能力的培养是大势所趋。本文以小组合作理论为基础, 探讨分析了加强 对大学生思辨能力培养的具体举措和如何评测大学生的思辨能力, 试图总结 出一条大学英语思辨能力教学的有效路径。

关键词: 小组合作, 《综合英语》, 思辨能力 